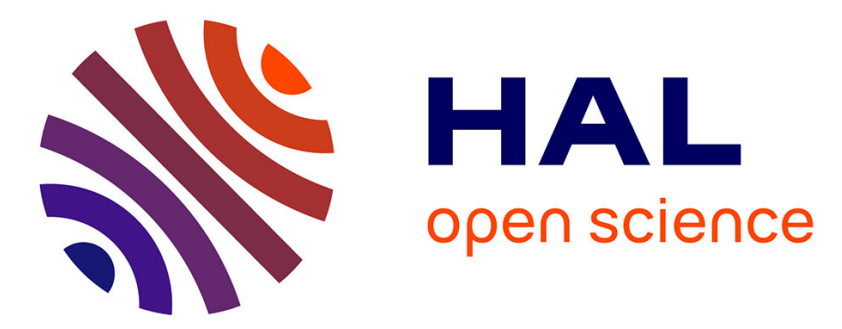

\title{
FROM LOCAL CLASS FIELD TO THE CURVE AND VICE VERSA
}

Laurent Fargues

\section{To cite this version:}

Laurent Fargues. FROM LOCAL CLASS FIELD TO THE CURVE AND VICE VERSA. AMS

Summer Institute in Algebraic Geometry, Jul 2015, Salt Lake City, United States. hal-01216763v2

\section{HAL Id: hal-01216763 \\ https://hal.science/hal-01216763v2}

Submitted on 11 Feb 2017

HAL is a multi-disciplinary open access archive for the deposit and dissemination of scientific research documents, whether they are published or not. The documents may come from teaching and research institutions in France or abroad, or from public or private research centers.
L'archive ouverte pluridisciplinaire $\mathbf{H A L}$, est destinée au dépôt et à la diffusion de documents scientifiques de niveau recherche, publiés ou non, émanant des établissements d'enseignement et de recherche français ou étrangers, des laboratoires publics ou privés. 


\title{
FROM LOCAL CLASS FIELD TO THE CURVE AND VICE VERSA
}

\author{
LAURENT FARGUES
}

\begin{abstract}
We begin by reviewing our joint work with J.-M. Fontaine about the fundamental curve of $p$-adic Hodge theory. We then explain our results obtained in 4 about the classification of $G$-bundles on this curve and its link with local class field theory. We finish by formulating conjectures that would extend those results.
\end{abstract}

\section{Contents}

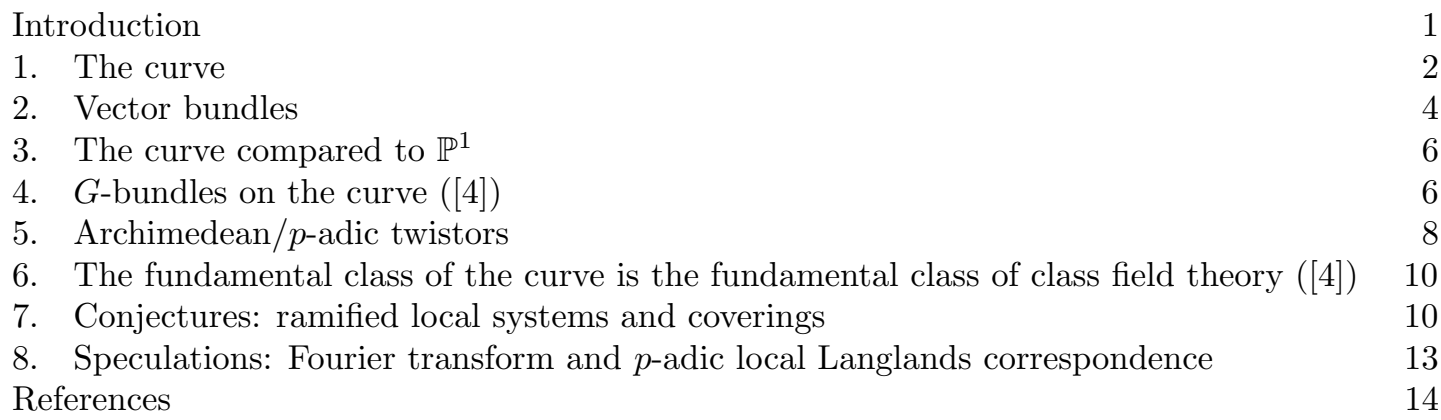

\section{INTRODUCTION}

Following work of Tate (28]) Fontaine developed the domain of $p$-adic Hodge theory by introducing the so-called $p$-adic period rings (see [10 for example). In our joint work with Fontaine ([7], [8]) we introduced a new object called "the curve". This has different incarnations. One of those incarnations is a Dedekind scheme over the p-adic numbers that, although not being of finite type, shares a lot of properties with "classical" proper smooth algebraic curves over a field.

A lot of the classical objects showing up in p-adic Hodge theory have an interpretation on the curve. For example Fontaine's period ring $\mathrm{B}_{d R}$ shows up as the completion of the local ring of this curve at a closed point at infinity. Fontaine's filtered $\varphi$-modules, some $p$-adic analog of archimedean Hodge structures, give rise to vector bundles on this curve. This has allowed us to reprove some important results of $p$-adic Hodge theory by studying vector bundles and their modifications on it. In some sense it is an analog of the twister projective line used in archimedean Hodge theory (see section 5).

In this article we first review the basic properties of this object and vector bundles on it. We then explain some more recent results obtained by the author in [4] about the study of $G$-bundles on the curve for $G$ a reductive group, and local class field theory.

Date: February 3, 2017.

L'auteur a bénéficié du support du projet ANR-14-CE25 "PerCoLaTor". 
Thanks: the author would like to thank Kiran Kedlaya and Martin Olsson for giving him the opportunity to talk about this subject at the AMS 2015 Summer Research Institute on Algebraic Geometry and giving him the occasion to write this report.

\section{THE CURVE}

Let $E$ be a finite extension of $\mathbb{Q}_{p}$ with residue field $\mathbb{F}_{q}$ and uniformizing element $\pi$. Let $F \mid \mathbb{F}_{q}$ be a complete non-archimedean perfect field, for example $\mathbb{F}_{q}\left(\left(T^{1 / \infty}\right)\right)$ or $\overline{\mathbb{F}_{q}((T))}$. In our joint work with Fontaine ([7) we have associated to this datum a "curve" that has two incarnations:

- The "algebraic" curve $X$, a one dimensional noetherian regular scheme of dimension 1 over $E$ (that is to say the gluing of a finite set of spectra of Dedekind rings)

- The "adic" curve $X^{a d}$ that is in a sense the $p$-adic Riemann surface associated to $X$.

Both are not of finite type over $E$ in the schematical sense for $X$ or in the topological sense for $X^{a d}$. Nonetheless, they satisfy striking properties analogous to the ones satisfied by proper smooth algebraic curve, resp. compact Riemann surfaces.

1.1. The adic curve. The algebraic curve was discovered first but it is now easier to present the adic one first. For this consider

$$
Y=\operatorname{Spa}\left(W_{\mathcal{O}_{E}}\left(\mathcal{O}_{F}\right), W_{\mathcal{O}_{E}}\left(\mathcal{O}_{F}\right)\right) \backslash V\left(\pi\left[\varpi_{F}\right]\right)
$$

where $\varpi_{F} \in F$ satisfies $0<\left|\varpi_{F}\right|<1$. This is an adic space over $E$ in Huber's sense ([15]) that one may think of as being a Stein p-adic Riemann surface that is an open punctured disk where the variable is $\pi$ and the coefficients of the holomorphic functions are in $F$. More precisely consider

$$
W_{\mathcal{O}_{E}}\left(\mathcal{O}_{F}\right)=\left\{\sum_{n \geq 0}\left[x_{n}\right] \pi^{n} \mid x_{n} \in \mathcal{O}_{F}\right\},
$$

the ramified Witt vectors of $\mathcal{O}_{F}$ (the usual Witt vectors when $E=\mathbb{Q}_{p}$ ). The preceding Teichmüller power series expansion is unique (here we used that the $\operatorname{ring} \mathcal{O}_{F}$ is perfect to have such a description of the Witt vectors). One may consider

$$
\mathcal{O}(Y)^{b}:=W_{\mathcal{O}_{E}}\left(\mathcal{O}_{F}\right)\left[\frac{1}{\pi}, \frac{1}{\left[\varpi_{F}\right]}\right]=\left\{\sum_{n \gg-\infty}\left[x_{n}\right] \pi^{n}\left|x_{n} \in F, \sup _{n}\right| x_{n} \mid<\infty\right\}
$$

that is the algebra of holomorphic functions on $Y$ meromorphic at $\pi=0$ (the origin of our "punctured" disk) and bounded near the radius 1 (the "exterior circle" of our "radius 1 open punctured disk"). For any radius $\rho \in] 0,1\left[\right.$ there is an associated Gauss norm $|\cdot|_{\rho}$ on $\mathcal{O}(Y)^{b}$ ("the supremum norm" of an holomorphic function on the annulus with radius $\rho$ ) defined by

$$
\left|\sum_{n}\left[x_{n}\right] \pi^{n}\right|_{\rho}=\sup _{n}\left|x_{n}\right| \rho^{n}
$$

By definition, the Frechet algebra of holomorphic functions on $Y$ is

$$
\mathcal{O}(Y)=\text { completion of } \mathcal{O}(Y)^{b} \text { with respect to }\left(|\cdot|_{\rho}\right)_{\rho \in] 0,1[} \text {. }
$$

The space $Y$ being Stein, $\mathcal{O}(Y)$ determines completely the space $Y$ itself.

There is a Frobenius $\varphi$ acting on the Witt vectors given by

$$
\varphi\left(\sum_{n}\left[x_{n}\right] \pi^{n}\right)=\sum_{n}\left[x_{n}^{q}\right] \pi^{n} .
$$

It extends to an action on $\mathcal{O}(Y)$ inducing an automorphism $\varphi$ of $Y$. This maps the annulus with radius $\rho$ to the annulus with radius $\rho^{1 / q}$ (since $\varphi$ raises the coefficients to the $q$-power and leaves invariant the variable $\pi$ it is an arithmetic Frobenius and thus dilates compared to a geometric Frobenius that would contract like in [11]). The action of $\varphi$ on $Y$ is thus totally discontinuous and we set

$$
X^{a d}:=Y / \varphi^{\mathbb{Z}} .
$$

One important point is the following. We put an index $E$ when we want so specify we use one of the spaces constructed using the field $E$. Let $E^{\prime} \mid E$ be a finite unramified extension of degree $h$ 
whose residue field is contained in $F$. Since $Y_{E}=Y_{E^{\prime}}$ (the ramified Witt vectors showing up in the definition of $Y_{E}$ do not change under an unramified change of $E$ ) is defined over $E^{\prime}$ one has

$$
Y_{E} \otimes_{E} E^{\prime}=\coprod_{\operatorname{Gal}\left(E^{\prime} \mid E\right)} Y_{E^{\prime}}
$$

where the Frobenius $\varphi_{E}$ permutes transitively the different components and $\varphi_{E^{\prime}}=\varphi_{E}^{h}$. From this one deduces that the finite Galois cover

$$
X_{E^{\prime}}^{a d}=X_{E}^{a d} \otimes_{E} E^{\prime} \longrightarrow X_{E}^{a d}
$$

can be identified with the unfolding morphism

$$
Y_{E} / \varphi^{h \mathbb{Z}} \longrightarrow Y_{E} / \varphi^{\mathbb{Z}}
$$

In an another direction, if $E^{\prime} \mid E$ is a complete perfectoid extension of $E$ then $X_{E}^{a d} \hat{\otimes}_{E} E^{\prime}$ is a perfectoid space $([24])$. We thus have the following picture:

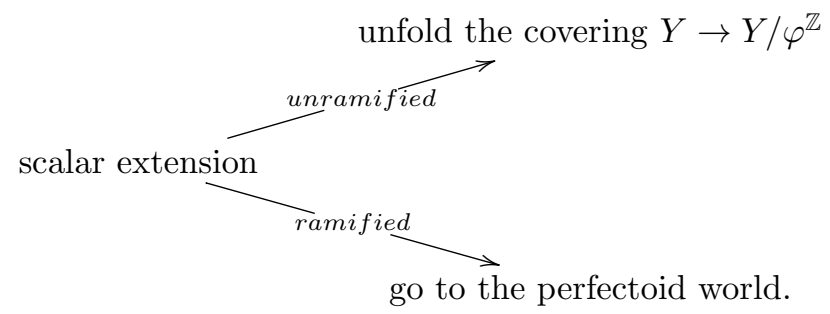

1.2. The algebraic curve. There is a natural line bundle $\mathcal{O}(1)$ on $X^{\text {ad }}$ whose pullback to $Y$ is trivial and given by the automorphy factor $\varphi \mapsto \pi^{-1}$. Its geometric realization is

$$
Y \underset{\varphi^{\mathbb{Z}}}{\mathbb{A}^{1} \longrightarrow Y / \varphi^{\mathbb{Z}}}
$$

where $\varphi$ acts on $\mathbb{A}^{1}$ by multiplication by $\pi^{-1}$. We set

$$
P=\bigoplus_{d \in \mathbb{N}} H^{0}\left(X^{a d}, \mathcal{O}(d)\right)
$$

as a graded algebra where

$$
\begin{aligned}
H^{0}\left(X^{a d}, \mathcal{O}(d)\right) & =\mathcal{O}(Y)^{\varphi=\pi^{d}} \\
& =\left\{f \in \mathcal{O}(Y) \mid \varphi(f)=\pi^{d} f\right\} .
\end{aligned}
$$

One has $P_{0}=H^{0}\left(X^{a d}, \mathcal{O}_{X^{a d}}\right)=E$,

$$
P_{1}=\left\{\sum_{n \in \mathbb{Z}}\left[x^{q^{-n}}\right] \pi^{n}|x \in F,| x \mid<1\right\},
$$

but the $E$-Banach spaces $P_{d}$ for $d \geq 2$ are more complicated (contrary to $P_{1}$ their elements do not have à priori a Laurent expansion around $\pi=0$ in general). Nevertheless those Banach spaces are finite dimensional in some extended sense defined by Colmez ([2]).

We now declare $\mathcal{O}(1)$ is ample and set

$$
X=\operatorname{Proj}(P) .
$$

One of the major discoveries of [7] is that this is a one dimensional noetherian regular scheme. This has profound consequences in $p$-adic Hodge theory, one of the main ones being the following. Suppose $F=\mathbb{C}_{p}^{b} \simeq \overline{\overline{\mathbb{F}_{p}((T))}}$. Then $X$ is canonically equipped with a closed point $\infty \in X$ such that:

- if $\mathrm{B}_{\text {cris }}$ is Fontaine's ring of crystalline periods $([9])$

$$
\Gamma\left(X \backslash\{\infty\}, \mathcal{O}_{X}\right)=\mathrm{B}_{\text {cris }}^{\varphi=\mathrm{Id}},
$$

- if $\mathrm{B}_{d R}$ is Fontaine's field of de Rham periods then

$$
\widehat{\mathcal{O}}_{X, \infty}=\mathrm{B}_{d R}^{+} .
$$


The stunning fact now is that $\mathrm{B}_{\text {cris }}^{\varphi=\mathrm{Id}}$ is a principal ideal domain!

The link between the adic an the algebraic curve is given by a morphism of ringed spaces

$$
X^{a d} \longrightarrow X
$$

that reflects the fact that $X^{a d}$ is to be thought of as the analytification of $X$ in some generalized sense. For example:

- it induces a bijection

$$
\left|X^{a d}\right| c l \stackrel{\sim}{\longrightarrow}|X|
$$

between the "classical Tate points" of $X^{a d}$ and the closed points of $X$

- for $x^{a d} \mapsto x$ via the preceding bijection

$$
\widehat{\mathcal{O}}_{X^{a d}, x^{a d}} \stackrel{\sim}{\longrightarrow} \widehat{\mathcal{O}}_{X, x} .
$$

Finally let us note that the curve $X$ is "complete" in the following sense. Let $E(X)$ be the function field of $X$. Then for any $f \in E(X)^{\times}$one has

$$
\operatorname{deg}(\operatorname{div}(f))=0 \text {. }
$$

Here to define the degree of a divisor we should explain that we have a natural definition of the degree of a closed point of $X$. The definition is a little bit involved in general, let us just say that if $F$ is algebraically closed then the degree of any closed point is 1 . This completeness of $X$ is fundamental for the proof of the classification of vector bundles in theorem 2.1 that follows. In fact it allows us to define the degree of a vector bundle and prove that they have Harder-Narasimhan filtrations as in the "classical setting".

\section{VECTOR BUNDLES}

We suppose from now on that $F$ is algebraically closed.

Before going further let us say that $\operatorname{Pic}^{0}(X)=0$ and

$$
\operatorname{Pic}(X)=<[\mathcal{O}(1)]>\stackrel{\operatorname{deg}}{\sim} \mathbb{Z} .
$$

This is linked to the fact that the ring $\mathrm{B}_{\text {cris }}^{\varphi=I d}$ is principal (for $F$ non-algebraically closed the equivalent of this ring is only Dedekind and $\mathrm{Pic}^{0}(X)$ is non-zero in general).

Let $\overline{\mathbb{F}}_{q}$ be the algebraic closure of $\mathbb{F}_{q}$ in $F$ and $L=W_{\mathcal{O}_{E}}\left(\overline{\mathbb{F}}_{q}\right)_{\mathbb{Q}}=\widehat{E^{u n}}$ the completion of the maximal unramified extension of $E$. Let $\sigma=\varphi$ be the Frobenius of $L$. We denote

$$
\varphi-\operatorname{Mod}_{L}=\{(D, \varphi)\}
$$

the corresponding category of isocrystals where $D$ is a finite dimensional $L$-vector space and $\varphi$ a $\sigma$-linear automorphism of $D$. Dieudonné and Manin have given a slope classification of such objects. The space $Y$ is defined over $L$. Thanks to this we can construct a functor

$$
\begin{aligned}
\varphi-\operatorname{Mod}_{L} & \longrightarrow \operatorname{Bun}_{X^{a d}} \\
(D, \varphi) & \longmapsto \mathscr{E}(D, \varphi)^{a d}
\end{aligned}
$$

where the geometric realization of $\mathscr{E}(D, \varphi)^{\text {ad }}$ is

$$
Y \underset{\varphi^{\mathbb{Z}}}{\times} D \longrightarrow Y / \varphi^{\mathbb{Z}}
$$

The morphism $X^{a d} \rightarrow X$ induces a GAGA functor

$$
\operatorname{Bun}_{X} \longrightarrow \operatorname{Bun}_{X^{a d}}
$$

such that $\mathscr{E}(D, \varphi)^{a d}$ is the analytification of a vector bundle $\mathscr{E}(D, \varphi)$ on $X$ associated to the graded $P$-algebra

$$
\bigoplus_{d \geq 0} H^{0}\left(X^{a d}, \mathscr{E}(D, \varphi)^{a d}(d)\right)=\bigoplus_{d \geq 0}\left(D \otimes_{L} \mathcal{O}(Y)\right)^{\varphi \otimes \varphi=\pi^{d}}
$$


We have the following classification theorem that is one of the main results of [7].

Theorem 2.1 ([7]). The functor

$$
\varphi-\operatorname{Mod}_{L} \stackrel{\mathscr{E}(-)}{\longrightarrow} \operatorname{Bun}_{X}
$$

is essentially surjective.

Via the Dieudonné-Manin theorem one can translate this theorem in the following way. For each slope $\lambda \in \mathbb{Q}$ there is defined a slope $\lambda$ stable bundle

$$
\mathcal{O}(\lambda)
$$

on $X$. If $\lambda=\frac{d}{h}$ with $(d, h)=1$ and $E_{h} \mid E$ is the degree $h$ unramified extension, $\mathcal{O}(\lambda)$ is the push forward of $\mathcal{O}_{X_{E_{h}}}(d)$ to $X_{E}$ via the finite Galois covering $X_{E_{h}} \rightarrow X_{E}$.

The preceding theorem then says that any vector bundle on $X$ is isomorphic to

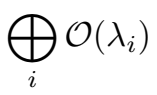

for a collection of slopes $\left(\lambda_{i}\right)_{i}$. Another way to state it in terms of semi-stability is the following two statements:

(1) Any semi-stable vector bundle of slope $\lambda$ is isomorphic to a direct sum of $\mathcal{O}(\lambda)$.

(2) The Harder-Narasimhan filtration of a vector bundle is split.

The main tools used to prove the preceding classification theorem are the following two deep statements about periods of $p$-divisible reinterpreted in terms of the curve:

(1) (Lafaille/Gross-Hopkins) Any modification $\mathscr{E} \hookrightarrow \mathcal{O}\left(\frac{1}{n}\right)$ of degree -1 of $\mathcal{O}\left(\frac{1}{n}\right)$ is such that $\mathscr{E} \simeq \mathcal{O}^{n}$, a trivial vector bundle.

(2) (Drinfeld) Any degree 1 modification $\mathcal{O}^{n} \hookrightarrow \mathscr{E}$ of $\mathcal{O}^{n}$ is such that $\mathscr{E} \simeq \mathcal{O}\left(\frac{1}{r}\right) \oplus \mathcal{O}^{n-r}$ for some $r$ satisfying $1 \leq r \leq n$.

Conversely, the preceding classification theorem can be used to obtain important informations about periods of $p$-divisible groups/periods of $p$-adic Galois representations in general (see for example chap. 10 of [7] and [26]). The curve/vector bundles on it together with the formalism of Harder-Narasimhan filtrations is a machinery that starts with the preceding two results as an input and recycles them by producing more general statements.

Finally let us remark that we have a GAGA theorem

$$
\operatorname{Bun}_{X} \stackrel{\sim}{\longrightarrow} \operatorname{Bun}_{X^{a d}}
$$

The GAGA functor and the preceding GAGA equivalence where first introduced and proven in ([6]) with the purpose of understanding the link between the preceding classification theorem and Kedlaya classification theorem of $\varphi$-modules over the so-called Robba ring associate to $F$ ([16]). In fact one checks using the expansion property of the action of $\varphi$ on $Y$ that $\operatorname{Bun}_{X^{a d}}$, that is to say $\varphi$-equivariant vector bundles on $Y$, are the same as germs of $\varphi$-equivariant vector bundles around the center $\pi=0$ of the "punctured disk" $Y$. Those last objects are exactly the $\varphi$-modules over the Robba ring. One then checks easily that Kedlaya's classification and the one of [7] match up via the GAGA functor. Kedlaya and Liu have since given a much more natural proof of GAGA in $([17])$ that allows us to deduce each classification theorem from the other one.

Finally let us note that there is more support to the fact that $X$ is "complete". In fact for any vector bundle $\mathscr{E}$ on $X$

$$
H^{0}(X, \mathscr{E}) \text { and } H^{1}(X, \mathscr{E}) \text { are finite dimensional...in the sense of Colmez ([2]). }
$$

We are going to look at an example to see what this means. For this let us pick a point $\infty \in|X|$ with residue field $C$. Then $C$ is a complete algebraically closed extension of $\mathbb{Q}_{p}$ (in general when $F$ is not algebraically closed those residue fields are perfectoid extensions of $\left.\mathbb{Q}_{p}\right)$. Fix $t \in H^{0}(X, \mathcal{O}(1))=P_{1}$ non-zero. There is an exact sequence

$$
0 \longrightarrow \mathcal{O}_{X} \stackrel{\times t}{\longrightarrow} \mathcal{O}_{X}(1) \longrightarrow i_{\infty *} C \longrightarrow 0 .
$$


Taking cohomology and using that $H^{1}\left(X, \mathcal{O}_{X}\right)=0$ (the curve has "genus 0 ", see the following section) one obtains an exact sequence

$$
0 \longrightarrow E \longrightarrow H^{0}(X, \mathcal{O}(1)) \longrightarrow C \longrightarrow 0 .
$$

This proves $H^{0}(X, \mathcal{O}(1))$ is an extension of a finite dimensional $C$-vector space by a finite dimensional $E$-vector space and thus is finite dimensional in Colmez sense. Let us note too that the preceding exact sequence is in fact nothing else than the so called fundamental exact sequence of p-adic Hodge theory ([9]) and this type of devissage of the cohomology of vector bundles sheds new light on it.

\section{The CURVE COMPARED TO $\mathbb{P}^{1}$}

There is a striking analogy between the classification theorem 2.1 and Grothendieck's classification of vector bundles on $\mathbb{P}^{1}([13])$. In fact it is interesting to note the following coincidences/discrepancies:

- Like $\mathbb{P}^{1}$, for any integer $d \geq 0$ one has $H^{1}(X, \mathcal{O}(d))=0$. In particular, $H^{1}\left(X, \mathcal{O}_{X}\right)=0$ and $X$ "has genus $0 "$.

- Contrary to $\mathbb{P}^{1}$ one has $H^{1}(X, \mathcal{O}(-1)) \neq 0$ (and it is even infinite dimensional over $E$ ).

In fact this last assertion can be reinterpreted in the following ways:

- Take a non-split extension

$$
0 \longrightarrow \mathcal{O} \longrightarrow \mathscr{E} \longrightarrow \mathcal{O}(1) \longrightarrow 0
$$

given by a non-zero class in $\operatorname{Ext}^{1}(\mathcal{O}(1), \mathcal{O})=H^{1}(X, \mathcal{O}(-1))$. Then one checks easily that necessarily the rank 2 vector bundle $\mathscr{E}$ is stable of slope $\frac{1}{2}$ and in fact is isomorphic to $\mathcal{O}\left(\frac{1}{2}\right)$.

- Pick a closed point $\infty \in|X|$ and denote

$$
\mathrm{B}_{e}:=\Gamma\left(X \backslash\{\infty\}, \mathcal{O}_{X}\right), \mathrm{B}_{d R}^{+}:=\widehat{\mathcal{O}}_{X, \infty} .
$$

Fix $t$ a uniformizing element of $\mathrm{B}_{d R}^{+}$. Cech cohomology computations around formal neighborhoods of $\infty$ lead to the formulas

$$
\begin{aligned}
H^{1}(X, \mathcal{O}) & =\mathrm{B}_{d R}^{+}\left[\frac{1}{t}\right] /\left(\mathrm{B}_{d R}^{+}+\mathrm{B}_{e}\right) \\
H^{1}(X, \mathcal{O}(-1)) & =\mathrm{B}_{d R}^{+}\left[\frac{1}{t}\right] /\left(t \mathrm{~B}_{d R}^{+}+\mathrm{B}_{e}\right) .
\end{aligned}
$$

Let us denote $\operatorname{deg}=-\operatorname{ord}_{\infty}: \mathrm{B}_{e} \rightarrow \mathbb{N} \cup\{-\infty\}$. The vanishing of the first cohomology group says exactly that $\left(\mathrm{B}_{e}, \mathrm{deg}\right)$ is almost euclidean like $\mathbb{P}^{1}$ in sense that for any $x, y \in \mathrm{B}_{e}$ with $y \neq 0$ there exists $a, b$ such that $x=a y+b$ with $\operatorname{deg}(b) \leq \operatorname{deg}(y)$. The non-vanishing of the second one says that $\left(\mathrm{B}_{e}, \mathrm{deg}\right)$ is non-euclidean contrary to $\mathbb{P}^{1}$.

We have in thus a set of "equivalent" properties ([7] sec.5.4) that make $X$ different from $\mathbb{P}^{1}$ :

(1) $H^{1}(X, \mathcal{O}(-1)) \neq 0$

(2) There exists a stable vector bundle on $X$ with non-integral slope.

(3) The almost euclidean ring $\left(\mathrm{B}_{e}, \mathrm{deg}\right)$ is non-euclidean.

Finally let us say that the non-vanishing of $H^{1}(X, \mathcal{O}(-1))$ makes the proof of the classification theorem 2.1 much more difficult than Grothendieck's for $\mathbb{P}^{1}$.

\section{G-BUndles ON THE CURVE ([4])}

4.1. Classification in terms of Kottwitz set. Let $G$ be a reductive group over $E$. There are two equivalent definitions of a $G$-bundle on $X$ :

(1) The Tannakian one: exact tensor functors

$$
\operatorname{Rep}(G) \longrightarrow \operatorname{Bun}_{X}
$$

where $\operatorname{Rep}(G)$ is the category of linear representations of $G$.

(2) The geometric one: $G$-torsors on $X$ locally trivial for the étale topology. 
To go from (1) to $(2)$ one associates to $\omega: \operatorname{Rep}(G) \rightarrow \operatorname{Bun}_{X}$ the torsor

$$
\operatorname{Isom}\left(\omega_{\text {can }}, \omega\right)
$$

where $\omega_{c a n}(V, \rho)=V \otimes_{E} \mathcal{O}_{X}$. To go from (2) to (1) one associates to $\mathscr{T}$ over $X$ the functor

$$
(V, \rho) \longmapsto \underset{\rho}{ } \underset{\rho}{\times} V .
$$

Recall Kottwitz set $([20])$

$$
\mathrm{B}(G)=G(L) / \sigma \text {-conjugacy. }
$$

If $b \in G(L)$ it defines an isocrystal with a $G$-structure

$$
\begin{aligned}
\operatorname{Rep}(G) & \longrightarrow \varphi-\operatorname{Mod}_{L} \\
(V, \rho) & \longmapsto\left(V_{L}, \rho(b) \sigma\right) .
\end{aligned}
$$

Composing with the functor

$$
\mathscr{E}(-): \varphi-\operatorname{Mod}_{L} \longrightarrow \operatorname{Bun}_{X}
$$

we obtain a $G$-bundle $\mathscr{E}_{b}$. The main theorem of 4 is now the following.

Theorem 4.1. The preceding construction induces a bijection of pointed sets

$$
\begin{aligned}
\mathrm{B}(G) & \stackrel{\longrightarrow}{\longrightarrow} H_{\text {ét }}^{1}(X, G) \\
{[b] } & \longmapsto
\end{aligned}
$$

This theorem has very nice features. Let us give some examples:

- There is an inclusion of Galois cohomology

$$
H^{1}(E, G) \subset \mathrm{B}(G)
$$

that identifies $H^{1}(E, G)$ with the subset of "unit root" (that is to say slope 0 ) $G$-isocrystals. At the level of $H^{1}(X, G)$ this is given by the pullback in étale cohomology along the structural morphism $X \rightarrow \operatorname{Spec}(E)$.

- When $G$ is quasi-split there is a good dictionary between Kottwitz description of the set $\mathrm{B}(G)$ and Atiyah-Bott reduction theory for $G$-bundles. For example: $b$ is basic if and only if $\mathscr{E} b$ is semi-stable.

4.2. The case of tori: class field theory shows up. The proof of theorem 4.1 is quite involved from the $p$-adic Hodge theoretic point of view. Nevertheless, after having proved this theorem, the author thought that maybe he should have found a simple proof for tori first. This has lead him finally to consider the links between the curve and class field theory. Here is how.

Let $T$ be torus over $E$. The map $\mathrm{B}(T) \rightarrow H^{1}(X, T)$ is a morphism of groups. Let $\mathcal{F}$ be a functor from the category of tori over finite extensions of $E$ toward abelian groups. According to Kottwitz (20] 2.2) a morphism $\mathrm{B}(-) \rightarrow \mathcal{F}$ is an isomorphism if and only if:

(1) one has $\mathcal{F}\left(\mathbb{G}_{m}\right)=\mathbb{Z}$,

(2) for $E^{\prime} \mid E$ finite one has an identification $\mathcal{F}\left(\operatorname{Res}_{E^{\prime} / E} T\right)=\mathcal{F}(T)$,

(3) The functor $\mathcal{F}$ is exact on the right.

For $H^{1}(X,-)$ property $(1)$ is easily satisfied since $\operatorname{Pic}(X)=<[\mathcal{O}(1)]>$. Property (2) is evident since, if $f: X_{E^{\prime}} \rightarrow X_{E}, H^{1}\left(X_{E^{\prime}}, T\right)=H^{1}\left(X_{E}, f_{*} T\right)$. To prove property (3) one is naturally lead to the following theorem.

Theorem 4.2. For $T$ a torus over $E$ on has $H^{2}(X, T)=0$.

A dévissage using an Hochschild-Serre spectral sequence for a finite extension of $E$ shows that it suffices to proves the result for a split torus, that is to say, up to replacing $E$ by a finite extension, the following theorem.

Theorem 4.3. On has $H^{2}\left(X, \mathbb{G}_{m}\right)=0$. 
Thanks to Grothendieck ([14 2.2) this cohomological Brauer group can be identified with the Brauer group

$$
\operatorname{Br}(X)=\{\text { Azumaya algebras over } X\} / \sim .
$$

We are thus lead to prove the following theorem.

Theorem 4.4. On has $\operatorname{Br}(X)=0$.

Here is the key proposition used to prove this theorem. This is where class field theory shows up in an unexpected way.

Proposition 4.5 (Key proposition). The morphism $\operatorname{Br}(E) \rightarrow \operatorname{Br}(X)$ is zero.

Proof. Let B be a simple $E$-algebra. Local class field theory (computation of $\operatorname{Br}(E)$ ) says that there exists an isoclinic isocrystal $(D, \varphi) \in \varphi-\operatorname{Mod}_{L} \operatorname{such}$ that $\mathrm{B} \simeq \operatorname{End}(D, \varphi)$. Then one checks easily that by applying the functor $\mathscr{E}(-)$ one has

$$
\mathrm{B} \otimes_{E} \mathcal{O}_{X} \stackrel{\sim}{\longrightarrow} \mathscr{E} n d(\mathscr{E}(\mathrm{D}, \varphi))
$$

The remaining part of the proof of theorem 4.4 goes the following way. The $\mathrm{PGL}_{n}$-torsor associated to an Azumaya algebra of rank $n^{2}$ on $X$ has a canonical Atiyah-Bott reduction to a parabolic subgroup. More precisely, for such an Azumaya algebra $\mathcal{A}$ its Harder-Narasimhan filtration $\left(\mathcal{A}^{\geq \lambda}\right)_{\lambda \in \mathbb{Q}}$ (part of the filtration where the H.N.-slope is greater than or equal to $\lambda$ ) satisfies properties such as $\mathcal{A}^{\geq \lambda} \cdot \mathcal{A}^{\geq \mu} \subset \mathcal{A}^{\geq \lambda+\mu}$. One then checks that the datum given by the sub-algebra $\mathcal{A}^{\geq 0}$ defines such a reduction.

Using a statement analogous to the fact that the Harder-Narasimhan filtration of a vector bundle is split, one then proves that this has in fact a reduction to a semi-stable torsor of a Levi subgroup of $\mathrm{PGL}_{n}$. In terms of the preceding notations this semi-stable torsor is associated to the algebra $\mathcal{A}^{\geq 0} / \mathcal{A}^{>0}$. Now, thanks to theorem 4.1 there is a $\otimes$-equivalence

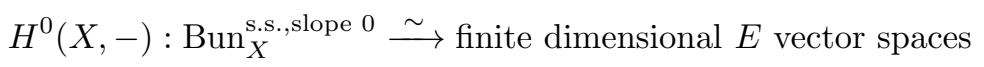

whose inverse is given by $V \mapsto V \otimes_{E} \mathcal{O}_{X}$. We deduce from this that if $\mathscr{B}$ is a slope 0 semi-stable Azumaya algebra then $\mathscr{B}=\mathrm{B} \otimes_{E} \mathcal{O}_{X}$ with B a simple $E$-algebra. We can then apply proposition 4.5 to conclude.

4.3. Vice Versa. We have seen that local class field theory implies theorem 4.1 for tori. Conversely, suppose theorem 4.1 known. Since the map B $\left(\mathrm{GL}_{n}\right) \rightarrow \mathrm{B}\left(\mathrm{PGL}_{n}\right)$ is surjective we deduce the vanishing theorem 4.4 and thus in particular proposition 4.5 . Reversing the proof of proposition 4.5 and using the classification theorem for vector bundles we deduce a new proof of local class field theory. Of course, this is probably the most complicated proof ever given of local class field theory. That being said, it is quite natural and tells us that the vanishing theorem 4.4 really encodes local class field theory.

\section{ArchimedeAn $/ p$-ADIC TWISTORS}

Let

$$
\widetilde{\mathbb{P}}_{\mathbb{R}}^{1}=\mathbb{P}_{\mathbb{C}}^{1} / z \sim-\frac{1}{\bar{z}}
$$

be the Twistor projective line over $\mathbb{R}$ that is to say the real quadric without real point or the Severi-Brauer variety associated to Hamilton quaternions over $\mathbb{R}$. In the preceding notation $\mathbb{P}_{\mathbb{C}}^{1}$ is considered as a scheme over $\mathbb{R}$ by restriction of scalars. It seems like this is the analog of our curve $X$ for $E=\mathbb{R}$, the analogue for $E=\mathbb{C}$ being $\mathbb{P}_{\mathbb{C}}^{1}$.

Over $\mathbb{C}$ we have Grothendieck's classification of vector bundles that looks like classification theorem 2.1. More generally, for a reductive group $G$ over $\mathbb{C}$, we have a bijection

$$
\begin{aligned}
\mathrm{B}(G) & \stackrel{\longrightarrow}{\longrightarrow} H^{1}\left(\mathbb{P}_{\mathbb{C}}^{1}, G\right) \\
{[\nu] } & \longmapsto
\end{aligned}
$$


where $\mathrm{B}(G)$ is Kottwitz set of algebraic morphisms $\mathbb{C}^{\times} \rightarrow G(\mathbb{C})$ up to $G(\mathbb{C})$-conjugation $([19],[13])$.

Over $\mathbb{R}$ the classification of vector bundles over $\widetilde{\mathbb{P}}_{\mathbb{R}}^{1}$ is remarkably analogous to theorem 2.1 . More precisely, denote

$$
u: \mathbb{P}_{\mathbb{C}}^{1} \rightarrow \widetilde{\mathbb{P}}_{\mathbb{R}}^{1}
$$

a $\mathbb{Z} / 2 \mathbb{Z}$-Galois covering. One defines for each slope $\lambda \in \frac{1}{2} \mathbb{Z}$ a vector bundle $\mathcal{O}(\lambda)$ on $\widetilde{\mathbb{P}}_{\mathbb{R}}^{1}$ such that:

- if $\lambda \notin \mathbb{Z}$ then $\mathcal{O}(\lambda)=u_{*} \mathcal{O}_{\mathbb{P}_{\mathbb{C}}^{1}}(2 \lambda)$,

- if $\lambda \in \mathbb{Z}$ then $u^{*} \mathcal{O}(\lambda)=\mathcal{O}_{\mathbb{P}_{\mathbb{C}}^{1}}(2 \lambda)$.

Then one checks easily that the exact analogue of theorem 2.1 holds for $\widetilde{\mathbb{P}}_{\mathbb{R}}^{1}$. More generally, if $G$ is reductive over $\mathbb{R}$, we have the following proposition.

Proposition 5.1. There is a bijection $\mathrm{B}(G) \stackrel{\sim}{\longrightarrow} H^{1}\left(\widetilde{\mathbb{P}}_{\mathbb{R}}^{1}, G\right)$.

Here $\mathrm{B}(G)=H_{\text {alg }}^{1}\left(W_{\mathbb{R}}, G(\mathbb{C})\right)$ is Kottwitz set of equivalence classes of cocycles $W_{\mathbb{R}} \rightarrow G(\mathbb{C})$ (where the Weil group $W_{\mathbb{R}}$ acts through its quotient $\operatorname{Gal}(\mathbb{C} \mid \mathbb{R})$ ) whose restriction to $\mathbb{C}^{\times}$is algebraic (19]). The proof of the preceding proposition is an exercise in descent theory along $u$. In fact, $W_{\mathbb{R}}=\mathbb{C}^{\times} \cup \mathbb{C}^{\times} \tilde{c}$ where $\tilde{c}$ is a lift of complex conjugation satisfying $\tilde{c}^{2}=-1$. Moreover, if $f(z)=-\frac{1}{\bar{z}}$ there is an isomorphism $v: f^{*} \mathcal{O}_{\mathbb{P}_{\mathbb{C}}^{1}}(1) \stackrel{\sim}{\longrightarrow} \mathcal{O}_{\mathbb{P}_{\mathbb{C}}^{1}(1)}(1)$ satisfying

$$
v \circ f^{*} v=-1 \text {. }
$$

A cocycle computation then shows that

$$
\begin{aligned}
H_{\mathrm{alg}}^{1}\left(\mathbb{C}^{\times} \rtimes \operatorname{Gal}(\mathbb{C} \mid \mathbb{R}), G(\mathbb{C})\right) & \stackrel{\sim}{\longrightarrow} H^{1}\left(\mathbb{P}_{\mathbb{R}}^{1}, G\right) \\
H_{\mathrm{alg}}^{1}\left(W_{\mathbb{R}}, G(\mathbb{C})\right) & \stackrel{\sim}{\longrightarrow} H^{1}\left(\widetilde{\mathbb{P}}_{\mathbb{R}}^{1}, G\right) .
\end{aligned}
$$

\begin{tabular}{|c|c|}
\hline Hodge theory & $p$-adic Hodge theory \\
\hline $\begin{array}{l}\text { Vector bundles on } \widetilde{\mathbb{P}}_{\mathbb{R}}^{1} \\
\mathbb{C}^{\times} \text {-vector bundles on } \widetilde{\mathbb{P}}_{\mathbb{p}}^{1}\end{array}$ & $\begin{array}{c}\text { Vector bundles on } X \\
\operatorname{Gal}(\bar{K} \mid K) \text {-equivariant vector bundles on } X\end{array}$ \\
\hline $\begin{array}{l}\text { Modification at } \infty \text { of } \mathbb{C}^{\times} \text {-v.b. } \mathscr{E}_{1} \text { to } \mathscr{E}_{2} \\
\text { on } \widetilde{\mathbb{P}}_{\mathbb{R}}^{1} \text { with } \mathscr{E}_{1} \text { s.s. of slope } 0\end{array}$ & $\left\{\begin{array}{l}\text { Modification at } \infty \text { of } \operatorname{Gal}(\bar{K} \mid K) \text {-v.b. } \mathscr{E}_{1} \text { to } \mathscr{E}_{2} \\
\text { on } X \text { with } \mathscr{E}_{1} \text { s.s. of slope } 0\end{array}\right.$ \\
\hline
\end{tabular}

Suppose $E=\mathbb{Q}_{p}$ and $F=\widehat{\bar{K}}^{b}$, in which case $X$ is equipped with an action of $\operatorname{Gal}(\bar{K} \mid K)$ stablizing a closed point $\infty$ whose residue field is $\widehat{\bar{K}}$.

For the last line:

- To any real Hodge structure on the real vector space $V$ there is associated such a modification with $\mathscr{E}_{1}=V \otimes_{\mathbb{R}} \mathcal{O}_{\widetilde{P}_{\mathbb{R}}^{1}}$ and the modification at $\infty$ is given by the Hodge filtration of $V_{\mathbb{C}}$ via the lattice $\mathrm{Fil}^{0}\left(V_{\mathbb{C}} \otimes \mathbb{C}((t))\right)$ where $\mathbb{C} \llbracket t \rrbracket=\widehat{\mathcal{O}}_{\mathbb{P}_{\mathbb{C}}^{1}}, \infty$, the modification at 0 is given by the complex conjugate of the Hodge filtration. This gives rises to a Twistor as defined by Simpson ([27]).

- To any de Rham Galois representation $V$ of $\operatorname{Gal}(\bar{K} \mid K)$ there is associated such a modification with $\mathscr{E}_{1}=V \otimes \mathcal{O}_{X}$ and the modification is given by the lattice $D_{d R}(V) \otimes_{K} \mathrm{~B}_{d R}^{+}$ where $D_{d R}(V)$ is the filtered $K$-vector space defined by Fontaine.

Such equivariant modifications of vector bundles on $X$ show up in a fundamental way in the chapter 10 of [7]. Finally let us point that in ([6]) the author gave a classification of such modification (without the Galois action) in terms of "Sthukas" that is to say in this context in terms of some Breuil-Kisin type $\varphi$-modules over the ring $W_{\mathcal{O}_{E}}\left(\mathcal{O}_{F}\right)$.

Recently ([1]) Bhatt, Morrow and Scholze have announced the construction of:

- a cohomology theory for proper smooth algebraic varieties having good reduction over $K$ producing such $\varphi$-modules, 
- a $q$-de Rham cohomology theory for proper smooth algebraic varieties over $\mathbb{Q}$ that should allow to interpolate between the two columns of the preceding array that is to say between the archimedean place and primes $p$ of good reduction.

\section{The Fundamental Class of The CURVE IS THE FUndamental Class of Class FiELD} THEORY ([4])

6.1. The fundamental class. Using the vanishing 4.3 of $H^{2}\left(X, \mathbb{G}_{m}\right)$, the computation of the Picard group of $X$ and Kummer exact sequence we obtain a trace isomorphism for any integer $n$

$$
\operatorname{tr}: H^{2}\left(X, \mu_{n}\right) \stackrel{\sim}{\longrightarrow} \mathbb{Z} / n \mathbb{Z}
$$

Let us denote

$$
\eta_{X}:=c_{1}(\mathcal{O}(1)) \in H^{2}\left(X, \mu_{n}\right)
$$

One has $\operatorname{tr}\left(\eta_{X}\right)=1$ and we call $\eta_{X}$ the fundamental class of $X$.

\section{2. Étale cohomology of local systems on the curve. One has the following theorem.}

Theorem 6.1 ([7] 8.6). The curve $X$ is geometrically simply connected that is to say any finite étale cover of $X \otimes_{E} \bar{E}$ has a section.

Let us denote $\Gamma_{E}=\operatorname{Gal}(\bar{E} \mid E)$. From this one deduces an equivalence between finite discrete $\Gamma_{E}$-modules and étale local systems on $X$. Now, working again with Kummer sequences and Hoschild-Serre spectral sequences we obtain the following theorem.

Theorem 6.2. Let $M$ be a finite discrete $\Gamma_{E}$-module and $\mathscr{F}$ the associated local system on $X_{\text {ét }}$. For $0 \leq i \leq 2$ one has an an isomorphism

$$
H^{i}\left(\Gamma_{E}, M\right) \stackrel{\sim}{\longrightarrow} H^{i}(X, \mathscr{F}) .
$$

The most striking fact is now the following. Via the preceding isomorphism

$$
\operatorname{Br}(E)[n]=H^{2}\left(E, \mu_{n}\right) \stackrel{\sim}{\longrightarrow} H_{\text {ét }}^{2}\left(X, \mu_{n}\right)
$$

the fundamental class of local class field corresponds to the fundamental class $\eta_{X}$ of the curve.

Another way to state it is the following. The fundamental class of class field theory in $\operatorname{Br}(E)[n]$ is the class of the gerb of fibre functors on the Tannakian category of isocrystals whose slopes lie in $\frac{1}{n} \mathbb{Z}$. This Tannakian category is a non-geometric algebraic object: it is defined by some tensor relations among generators of this Tannakian category. The preceding tells us that the pullback to $X$ of this gerb has a geometric interpretation: this is the gerb of $n$-roots of $\mathcal{O}(1)$. Via the isomorphism (1) this characterizes completely this gerb.

6.3. Geometric interpretation of some results of Tate in Galois cohomology. Theorem 6.2 allows us to give some nice interpretations of the following two results of Tate:

(1) Tate-Nakayama duality is nothing else than Poincaré duality in étale cohomology of $X$

(2) Tate's formula for the Euler-Poincaré characteristic in Galois cohomology is nothing else than the usual formula for the Euler-Poincaré characteristic of a local system on a curve (Grothendieck-Ogg-Shafarevich).

\section{Conjectures: RAmified local Systems AND COVERINGS}

The preceding results on Galois cohomology have lead us to a set of conjectures. 
7.1. The function field of the curve is (C1). Of course, theorem 4.4 would have been much easier if we had an analog of Tsen's theorem for $X$. In fact we conjecture the following.

Conjecture 7.1. The function field of $X$ is $(\mathrm{C} 1)$.

In terms of $p$-adic Hodge theory this function field is

$$
\operatorname{Frac}\left(\mathrm{B}_{\text {cris }}^{+}\right)^{\varphi=I d} \text {. }
$$

Of course, this is not of finite type over $E$. Note that since $X$ is geometrically connected the algebraic closure of $E$ in this function field is $E$ itself. Moreover one can deduce from theorem 4.4 the fact that the Brauer group of this function field is zero (4 2.5).

Let us remark that for $E=\mathbb{C}$ (see sec 5 ) the preceding conjecture is satisfied by Tsen. For $E=\mathbb{R}$ this is an old conjecture due to Lang (21] p.379).

7.2. Comparison with the étale cohomology of $X^{a d}$. The morphism $X^{a d} \rightarrow X$ induces a morphism of sites $X_{\text {ét }}^{a d} \rightarrow X_{\text {ét }}$. We thus pose the following conjecture.

Conjecture 7.2. For any torsion étale sheaf $\mathscr{F}$ on $X_{\text {ét }}$ there is an isomorphism

$$
H^{\bullet}(X, \mathscr{F}) \stackrel{\sim}{\longrightarrow} H^{\bullet}\left(X^{a d}, \mathscr{F}\right) .
$$

This conjecture should allow us to prove the vanishing of étale cohomology in degrees $>2$ for $X$. Moreover it should imply the fact that the function field of $X$ has cohomological dimension $\leq 1$

7.3. Local systems on open subsets and constructible sheaves. For $\mathscr{F} \in \mathbb{D}_{c}^{b}\left(X_{\text {ét }}, \mathbb{Z} / n \mathbb{Z}\right)$ let us set

$$
\mathbb{D}(\mathscr{F}):=R \mathscr{H} \text { om }(\mathscr{F}, \mathbb{Z} / n \mathbb{Z}(1)[2]) .
$$

It is then natural to hope for a generalization of Tate-Nakayama duality aka Poincaré duality for local systems.

Conjecture 7.3. For $\mathscr{F} \in \mathbb{D}_{c}^{b}\left(X_{\text {ét }}, \mathbb{Z} / n \mathbb{Z}\right)$ the pairing

$$
R \Gamma(X, \mathscr{F}) \times R \Gamma(X, \mathbb{D}(F)) \longrightarrow H^{2}(X, \mathbb{Z} / n \mathbb{Z}(1)) \underset{\operatorname{tr}}{\stackrel{\sim}{\longrightarrow}} \mathbb{Z} / n \mathbb{Z}
$$

is perfect.

This is reduced to proving that for $\mathscr{F}$ a $\mathbb{Z} / n \mathbb{Z}$-étale local system on an open subset $U$ of $X$ and $0 \leq i \leq 2$ the pairing

$$
H_{c}^{i}(U, \mathscr{F}) \times H^{2-i}\left(X, \mathscr{F}^{\vee}(1)\right) \longrightarrow H^{2}(X, \mathbb{Z} / n \mathbb{Z}(1)) \stackrel{\sim}{\longrightarrow} \mathbb{Z} / n \mathbb{Z}
$$

is perfect. Here by definition $H_{c}^{i}(U, \mathscr{F}):=H^{i}(X, j ! \mathscr{F})$ where $j: U \hookrightarrow X$.

There is a good category of étale torsion, resp. $\mathbb{Q}_{\ell}$, resp. $\mathbb{Q}_{p}$-perverse sheaves on $X$. Those categories of perverse sheaves are defined by the exact same formulas defining perverse sheaves on a "usual" smooth projective curve over a field (the fact that there is a t-structure on the corresponding bounded derived category with constructible cohomology needs a proof; one has to check that for $j: U \hookrightarrow X$ an open embedding and $\mathscr{F}$ a local system on $U$ then $R j_{*} \mathscr{F}$ is constructible, which is not evident but true). Let us denote $\Lambda \in\left\{\right.$ torsion, $\left.\mathbb{Q}_{\ell}, \mathbb{Q}_{\ell}\right\}$. There is an embedding

$$
\operatorname{Rep}_{\Lambda}\left(\Gamma_{E}\right) \subset \operatorname{Perv}_{\Lambda}(X) .
$$

where the left member is the category of local systems. This is compatible with Verdier duality where on $\operatorname{Rep}_{\Lambda}\left(\Gamma_{E}\right)$ Verdier duality is nothing else than Cartier duality $M \mapsto M^{\vee}(1)$. Conjecture 7.3 tells us that there should exist an extension of Tate-Nakayma duality to the cohomology of those perverse sheaves.

In particular, for almost everywhere unramified Galois representations of the function field of $X$, that is to say germs of local systems on non-empty open subsets $U$ of $X$, there exists a cohomology theory $\mathscr{F} \mapsto \mathbb{H} \bullet(U, \mathscr{F}):=H^{\bullet}\left(X, j_{*} \mathscr{F}\right)$ with $j: U \hookrightarrow X$, satisfying a genereralization of 
Tate-Nakayama duality.

Question 7.4. The local Langlands correspondence says there is a natural bijection

$$
\operatorname{Rep}_{\overline{\mathbb{Q}}_{\ell}}^{\text {Frob-s.s. }}\left(W_{E}\right) / \sim \simeq \coprod_{n \geq 1}\left\{\text { Irreducible smooth } \overline{\mathbb{Q}}_{\ell} \text {-representations of } \mathrm{GL}_{n}(E)\right\} / \sim .
$$

Is it possible to extend this to

$\{$ (finitely ramified) Weil sheaves on open subsets of $X\} / \sim \simeq\{$ Some unknown objects $\} / \sim ?$

From this perspective it would seem interesting to study ramified coverings of the curve. The author knows very little about their structure (Picard group, computation of $h^{1}(\mathcal{O})$, vector bundles on them...).

7.4. The fundamental group of an open subset. If $A$ is a profinite set we denote

$$
F(A)
$$

the profinite free-group on $A$. This means that if $G$ is a profinite group then

$$
\operatorname{Hom}(F(A), G)=\mathscr{C}(A, G)
$$

(continuous maps). Its topological abelianisation is

$$
F(A)^{a b}=\mathscr{C}(A, \widehat{\mathbb{Z}})
$$

Let

$$
U=X \backslash\left\{x_{1}, \ldots, x_{r}\right\}
$$

be an open subset of $X$. Let us remark that for $E^{\prime} \mid E$ finite, in the étale covering $X_{E^{\prime}} \rightarrow X_{E}$ each closed point is totally decomposed (this is due to the fact that the residue fields at closed points are algebraically closed since $F$ is algebraically closed itself). In particular in the pro-covering $f: X_{\bar{E}} \rightarrow X_{E}$ the fibre over a closed point $x$ of $X$ is isomorphic as a profinite set to

$$
f^{-1}(x) \simeq \operatorname{Hom}_{E}(\bar{E}, k(x)),
$$

a principal homogeneous space under $\Gamma_{E}$. For each $i, \widehat{\mathcal{O}}_{X, x_{i}}$ is isomorphic (non-canonically) to $k\left(x_{i}\right) \llbracket T \rrbracket$. One deduces that the $\pi_{1}$ of $\operatorname{Spec}\left(\widehat{\mathcal{O}}_{X, x_{i}}\left[\frac{1}{t_{i}}\right]\right)$ is isomorphic to $\widehat{\mathbb{Z}}(1)$. Let us denote

$$
A=\coprod_{i=1}^{r} \operatorname{Hom}_{E}\left(\bar{E}, k\left(x_{i}\right)\right) \simeq\{1, \ldots, r\} \times \Gamma_{E} .
$$

The morphism

$$
\coprod_{i=1}^{r} \operatorname{Spec}\left(\widehat{\mathcal{O}}_{X, x_{i}}\left[\frac{1}{t_{i}}\right]\right) \longrightarrow U
$$

then induces a morphism

$$
F(A) \longrightarrow \pi_{1}^{\text {géo }}(U)
$$

Since $X$ is geometrically connected this is surjective.

Conjecture 7.5. The preceding surjective morphism $F(A) \rightarrow \pi_{1}^{\text {géo }}(U)$ is an isomorphism.

We remark that this is true for $E=\mathbb{C}$ or $E=\mathbb{R}$ (see sec. 5). 
7.5. Anabelian interpretation of the fundamental class. One can check the following result using Gabber's cohomological absolute purity ([12]).

Theorem 7.6. Conjecture 7.5 is satisfied at the level of the abelianisation of the fundamental group:

$$
\bigoplus_{i=1}^{r} \mathscr{C}\left(\operatorname{Hom}_{E}\left(\bar{E}, k\left(x_{i}\right)\right), \widehat{\mathbb{Z}}(1)\right) \stackrel{\sim}{\longrightarrow} \pi_{1}^{g e o, a b}(U) .
$$

The $\pi_{1}^{a b}$ does not depend on the choice of a base point and the preceding theorem takes into account the action of $\Gamma_{E}$ independently of this choice of a base point. Now, suppose $r=1$. The extension given by the potentially abelian fundamental group of $U$ is

$$
1 \longrightarrow \pi_{1}^{g e o, a b}(U) \longrightarrow \pi_{1}(U) / \pi_{1}^{g e o}(U)^{d e r} \longrightarrow \Gamma_{E} \longrightarrow 1 \text {. }
$$

It is gives us a class in

$$
H^{2}\left(\Gamma_{E}, \mathscr{C}\left(\Gamma_{E}, \widehat{\mathbb{Z}}\right)(1)\right)=H^{2}\left(\Gamma_{E}, \widehat{\mathbb{Z}}(1)\right) .
$$

Conjecture 7.7. The class of the extension given by the potentially abelian fundamental group is given by the set of compatible fundamental classes of local class field theory in

$$
H^{2}\left(\Gamma_{E}, \widehat{\mathbb{Z}}(1)\right)={\underset{\leftarrow}{n}}_{\lim _{n}} \operatorname{Br}(E)[n] .
$$

\section{Speculations: Fourier transform and p-Adic local Langlands CORRESPondence}

The starting point is the following. Take $E=\mathbb{Q}_{p}$ and $F=\widehat{\overline{\mathbb{Q}}}_{p}^{b}$. The curve $X$ is then equipped with an action of $\operatorname{Gal}\left(\overline{\mathbb{Q}}_{p} \mid \mathbb{Q}_{p}\right)$. We then have two equivalences: the one we saw before

and the following

$$
\operatorname{Rep}_{\mathbb{Q}_{p}}\left(\operatorname{Gal}\left(\overline{\mathbb{Q}}_{p} \mid \mathbb{Q}_{p}\right)\right) \simeq \mathbb{Q}_{p} \text {-local systems on } X_{\text {ét }}
$$

$$
\begin{aligned}
\operatorname{Rep}_{\mathbb{Q}_{p}}\left(\operatorname{Gal}\left(\overline{\mathbb{Q}}_{p} \mid \mathbb{Q}_{p}\right)\right) & \stackrel{\sim}{\longrightarrow} \operatorname{Gal}\left(\overline{\mathbb{Q}}_{p} \mid \mathbb{Q}_{p}\right) \text {-equivariant slope } 0 \text { semi-stable vector bundles on } X \\
V & \longmapsto V \otimes_{\mathbb{Q}_{p}} \mathcal{O}_{X} .
\end{aligned}
$$

We thus deduce in an unnatural way an equivalence between the right sides of the two preceding equivalences. One can even check that this equivalence preserves cohomology, that is to say $\operatorname{Gal}\left(\overline{\mathbb{Q}}_{p} \mid \mathbb{Q}_{p}\right)$-equivariant cohomology of slope 0 semi-stable vector bundles corresponds to Galois cohomology.

The first question is the following:

- Is there a natural geometric way to describe directly the equivalence between étale $\mathbb{Q}_{p}$ local systems and equivariant slope 0 semi-stable bundle on $X$ ?

- Can it be extended to more general objects: for example $\mathbb{Q}_{p}$-perverse sheaves on one side and non-semi-stable equivariant vector bundles on the other side?

A "related" (the author's mind on this point is not clear at all) question is the following. Recall ([5]) that we have in terms of Scholze's diamonds ([25])

$$
X_{F}^{a d, \diamond}=\left(\operatorname{Spa}(F) \times \operatorname{Spa}\left(\mathbb{Q}_{p}\right)^{\diamond}\right) / \varphi^{\mathbb{Z}}
$$

where $\varphi$ is the Frobenius of the first factor $\operatorname{Spa}(F)$. We then have two structural maps

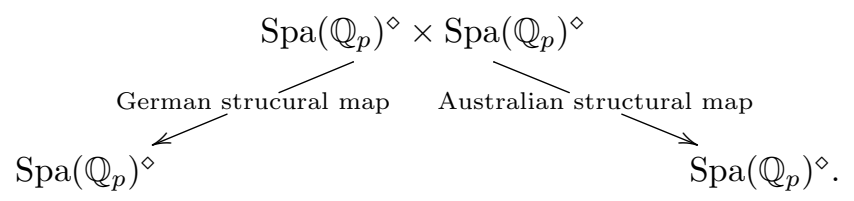

In terms of section 5 the left structural map is linked to the moduli spaces of $p$-divisible groups defined and studied by Rapoport and Zink ([23]). In their situation they look at $p$-divisible groups 
over changing bases. The right structural map is linked to moduli of $p$-divisible groups defined and studied by Kisin where the base is fixed but the coefficients vary ([18, 22]). Here is a set of questions:

(1) Is is possible to use this diagram to define a geometric Fourier transform ?

(2) Is there a way to exploit the automorphism of $\operatorname{Spa}\left(\mathbb{Q}_{p}\right)^{\diamond} \times \operatorname{Spa}\left(\mathbb{Q}_{p}\right)^{\diamond}$ defined by switching both factors?

Let us note that Kisin already remarked in [18 that the two types of spaces, the Australian one and the German one, seem to have the same type of singularities, which is a motivation for the preceding point (2).

This seems related to Colmez local Langlands correspondence ([3]) in the following way. There is an action

$$
\operatorname{Spa}\left(\mathbb{Q}_{p}^{c y c}\right)^{\diamond} \times \operatorname{Spa}\left(\mathbb{Q}_{p}^{c y c}\right)^{\diamond} \bigcirc N\left(\mathbb{Q}_{p}\right)
$$

where $N$ is the normalizer in $\mathrm{GL}_{2}\left(\mathbb{Q}_{p}\right)$ of the maximal split torus,

$$
N\left(\mathbb{Q}_{p}\right)=\left(\mathbb{Q}_{p}^{\times} \times \mathbb{Q}_{p}^{\times}\right) \rtimes \mathfrak{S}_{2} .
$$

Here to have an action of $\mathbb{Q}_{p}^{\times}$on $\operatorname{Spa}\left(\mathbb{Q}_{p}^{c y c}\right)^{\diamond}$ we use the action of $\mathbb{Z}_{p}^{\times}=\operatorname{Gal}\left(\mathbb{Q}_{p}^{c y c} \mid \mathbb{Q}_{p}\right)$ and make $p^{\mathbb{Z}}$ act as $\varphi^{\mathbb{Z}}$, that is to say

$$
\mathbb{Q}_{p}^{\times}=\operatorname{Gal}\left(\mathbb{Q}_{p}^{c y c} \mid \mathbb{Q}_{p}\right) \times \varphi^{\mathbb{Z}} .
$$

The main difficulty in the construction of [3] is to define an action of the non-trivial element of the Weyl group of $\mathrm{GL}_{2}$, which corresponds to this permutation of the two factors $\operatorname{Spa}\left(\mathbb{Q}_{p}\right)^{\diamond}$. Moreover, what Colmez does is, starting from a $(\varphi, \Gamma)$-module, to construct a Kirilov model of a representation of $\mathrm{GL}_{2}\left(\mathbb{Q}_{p}\right)$. The action of the non-trivial element of the Weyl group on Kirilov models is linked to epsilon factors...thus to Fourier transform. Once again, the author's mind on those problems is not clear at all, but let us point that for any $n$ the Weyl group of GL appears $_{n}$ as an automorphism group of the following product

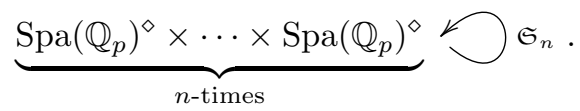

\section{REFERENCES}

[1] B. Bhatt, M. Morrow, and P. Scholze. Integral p-adic hodge theory - announcement. http://www.math.unibonn.de/people/scholze/IntPHodgeAnnouncement.pdf.

[2] P. Colmez. Espaces de Banach de dimension finie. J. Inst. Math. Jussieu, 1(3):331-439, 2002.

[3] P. Colmez. Représentations de $\mathrm{GL}_{2}\left(\mathbf{Q}_{p}\right)$ et $(\phi, \Gamma)$-modules. Astérisque, (330):281-509, 2010.

[4] L. Fargues. $G$-torseurs en théorie de Hodge $p$-adique. Prépublication.

[5] L. Fargues. Géométrisation de la correspondance de langlands locale. Prépublication.

[6] L. Fargues. Quelques résultats et conjectures concernant la courbe. In De la géométrie algébrique aux formes automorphes (I) - (Une collection d'articles en l'honneur du soixantième anniversaire de Gérard Laumon), volume 369 of Astérisque. 2015.

[7] L. Fargues and J.-M. Fontaine. Courbes et fibrés vectoriels en théorie de Hodge p-adique. Prépublication.

[8] L. Fargues and J.-M. Fontaine. Vector bundles on curves and p-adic hodge theory. In Automorphic Forms and Galois Representations, volume 415 of London Mathematical Society Lecture Note Series. Cambridge University Press, 2014.

[9] J.-M. Fontaine. Le corps des périodes p-adiques. Astérisque, (223):59-111, 1994. With an appendix by Pierre Colmez, Périodes $p$-adiques (Bures-sur-Yvette, 1988).

[10] J.-M. Fontaine. Représentations p-adiques semi-stables. Astérisque, (223):113-184, 1994. With an appendix by Pierre Colmez, Périodes $p$-adiques (Bures-sur-Yvette, 1988).

[11] K. Fujiwara. Rigid geometry, Lefschetz-Verdier trace formula and Deligne's conjecture. Invent.math., 127, 1997.

[12] K. Fujiwara. A proof of the absolute purity conjecture (after Gabber). In Algebraic geometry 2000, Azumino (Hotaka), volume 36 of Adv. Stud. Pure Math., pages 153-183. Math. Soc. Japan, Tokyo, 2002.

[13] A. Grothendieck. Sur la classification des fibrés holomorphes sur la sphère de Riemann. Amer. J. Math., 79:121-138, 1957.

[14] A. Grothendieck. Le groupe de Brauer. II. Théorie cohomologique. In Dix Exposés sur la Cohomologie des Schémas, pages 67-87. North-Holland, Amsterdam; Masson, Paris, 1968.

[15] R. Huber. A generalization of formal schemes and rigid analytic varieties. Math. Z., 217(no. 4):513-551, 1994.

[16] K. Kedlaya. A p-adic local monodromy theorem. Ann. of Math. (2), 160(1):93-184, 2004.

[17] K. Kedlaya and R. Liu. Relative p-adic Hodge theory: Foundations. Astérisque, 371, 2015. 
[18] M. Kisin. Moduli of finite flat group schemes, and modularity. Ann. of Math. (2), 170(3):1085-1180, 2009.

[19] R. Kottwitz. B(G) for all local and global fields. arXiv:1401.5728 [math.RT].

[20] R. Kottwitz. Isocrystals with additional structure. Compositio Math., 56(2):201-220, 1985.

[21] S. Lang. The theory of real places. Ann. of Math. (2), 57:378-391, 1953.

[22] G. Pappas and M. Rapoport. Ф-modules and coefficient spaces. Mosc. Math. J., 9(3):625-663, back matter, 2009.

[23] M. Rapoport and T. Zink. Period spaces for p-divisible groups. Number 141 in Annals of Mathematics Studies. Princeton University Press, Princeton, NJ, 1996.

[24] P. Scholze. Perfectoid spaces. Publ. Math. Inst. Hautes Études Sci., 116:245-313, 2012.

[25] P. Scholze. Notes du cours de P. Scholze à Berkeley. 2014.

[26] P. Scholze and J. Weinstein. Moduli of p-divisible groups. Camb. J. Math., 1(2):145-237, 2013.

[27] C. Simpson. Nonabelian Hodge theory. In Proceedings of the International Congress of Mathematicians, Vol. I, II (Kyoto, 1990), pages 747-756. Math. Soc. Japan, Tokyo, 1991.

[28] J. T. Tate. p-divisible groups.. In Proc. Conf. Local Fields (Driebergen, 1966), pages 158-183. Springer, Berlin, 1967.

Laurent Fargues, CNRS, Institut de Mathématiques de Jussieu, 4 Place Jussieu 75252 Paris

E-mail address: laurent.fargues@imj-prg.fr 